\title{
Spectral Clustering and Integration: The inner dynamics of computational geometry and spatial morphology
}

\author{
Tasos Varoudis and Alan Penn \\ Bartlett School of Architecture, University College London (UCL), London, UK \\ t.varoudis / a.penn [ [ucl.ac.uk]
}

\begin{abstract}
Deviating from common evaluation strategies of spatial networks that are realised through numerical comparison of single floating-point numbers such as global and local space syntax measures (centralities, connectivity, etc.) we aim to present a new computational methodology for creating detailed topo-geometric encodings of spaces that encapsulate some of the fundamental ideas about spatial morphology by Hillier (2007). In most cases, space syntax measures try to capture a particular quality of the space for comparison but they lose much of the detail of the spatial topo-geometry and morphology by mainly aggregating graph path traversals and not retaining any other information.

This research explores the use of weighted graph spectra, in a composite form, for the purpose of characterising the spatial structure as a whole. The new methodology focuses on the three primary space syntax graph modelling concepts, 'angular', 'metric' and 'topological', from the point of view of the resulting spatial geometries and develops new computational innovations in order to map spatial penetration of local neighbourhood spectra in different scales, dimensions and built environment densities in a continues way. The result is a new composite vector of high dimensionality that can be easily measured against others for detailed comparison. The proposed methodology is then demonstrated with the complete road-network dataset of Great Britain. The main dataset together with subsets is then used in a series of unsupervised machine learning analyses, including clustering and a form of Euclidian 'spectral integration'.
\end{abstract}

Keywords: graph analysis, graph spectra, unsupervised clustering, space syntax, spectral integration.

\section{Introduction}

In the space syntax field of research the evaluation of spatial networks is done using numerical comparison of streets or space with scalar measures such as graph centralities or connectivity. Through various graph types, including, axial and segment graphs (Hillier \& Hanson 1984; Turner 2005) there have been a number of effective representations of built environment that try to capture the particular quality of the space. These techniques though fail to retain much of the detail of the spatial topo-geometry and morphology by mainly aggregating graph path traversal measures. This is particularly discouraging if the aim is to employ machine learning techniques in your analysis of 
spatial dynamics. In space syntax practice, the overall understanding and classification of space based in a combination of secondary data, with spatial analysis measures representing only a very small number of data features in the analytical model.

This research explores the use of a new form of graph spectra (Robles-Kelly and Hancock 2003, Hanna 2009) in order to characterise the spatial structure as a whole. The new methodology presented here creates a unique composite spectra following on the three primary space syntax graph modelling concepts, 'angular', 'metric' and 'topological'. With this new computational model we aim to map spatial penetration of local neighbourhood spectra in different scales and dimensions focusing purely on geometric features. The result is a vector of high dimensionality that can be easily measured against others for detailed comparison. This feature space is then used in a series of unsupervised learning analyses building the first GB wide spatial model of this kind. The project uniquely achieves full scale analyses of Great Britain where large metropolitan and regional areas' detailed characteristics of the special morphology give intriguing results. Ultimately the focus of this stream of work is to develop novel approaches for the next generation of space syntax driven machine learning systems.

\section{From space syntax to multi-dimensional datascapes}

This project represents a set of steps in the direction of creating computational and research ground for space syntax theory and methodology towards machine intelligence driver models. While on first read the methodological and theoretical approach seems completely different, the aim is to reinvent the foundational building block of geometry and spatial configuration while linking to the ideas about built environment morphology pioneered in space syntax by Bill Hillier.

Space syntax (Hillier 2007) is a set of techniques for analysing spatial layouts and human activity patterns in buildings and urban areas. Some of the basic ideas proposed in the literature is that space is not a background to human activity, but is intrinsic to it and that space is first and foremost configurational (Hillier and Vaughan, 2007). That is to say, what happens in any individual space is fundamentally influenced by the relationships between that space and the network of spaces to which it is connected. Elements of space are considered geometrically derived like an axial line, segment or an isovist polygon area. In this project we look at GB wide road network models thus the concept of space syntax's street segment lines is used (Turner 2007). Relationships between street elements result from their configuration. These relationships can be objectively analysed using various graph-based measures (Varoudis 2012 - open source depthmapX, Turner 2007) were the graph represents the configurational relations between those spatial elements (example in figure 1). 


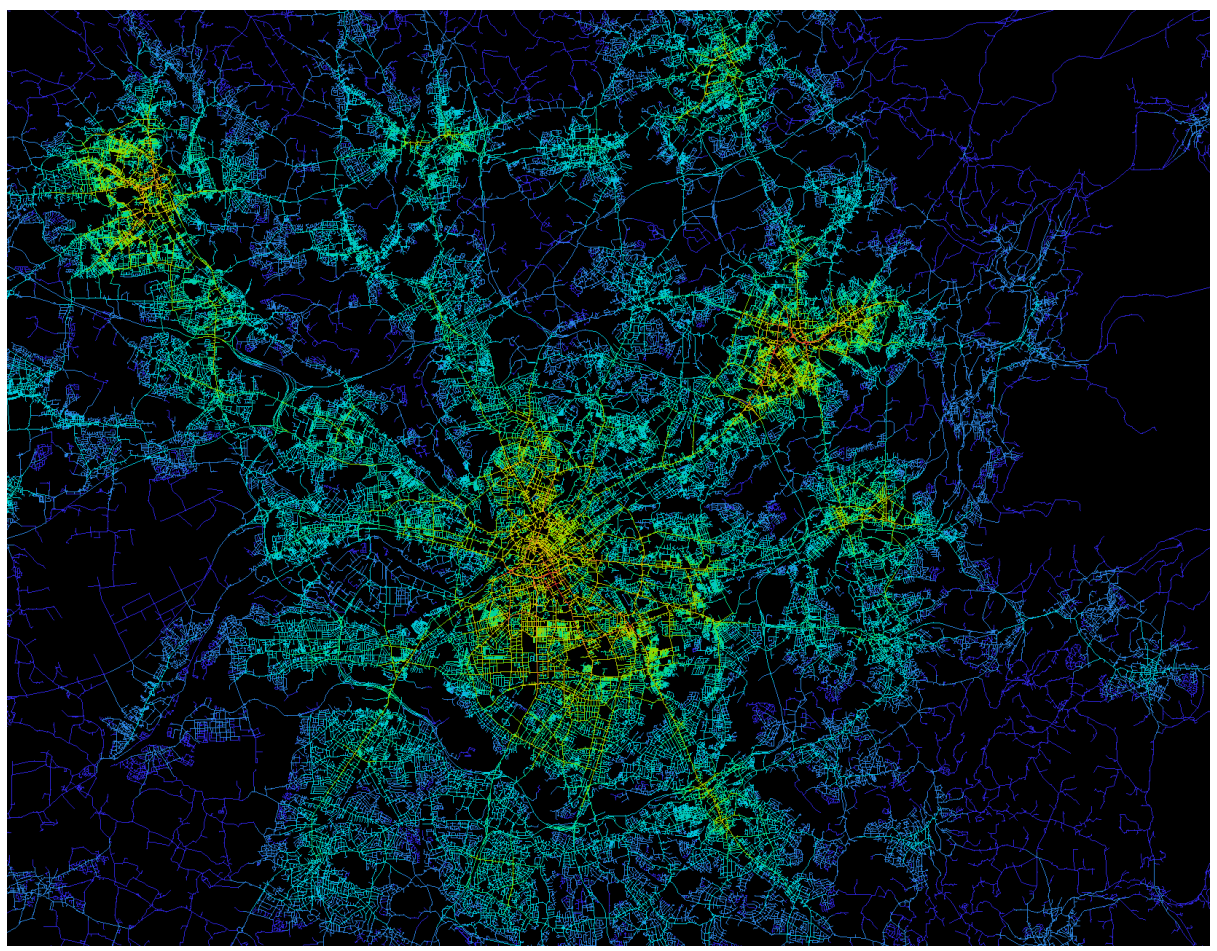

Fig. 1. Greater Manchester road network - space syntax analysis of integration or closeness centrality in graph sciences. Red is high, blue colour is low values.

Moreover, space syntax heavily relays on three basic ways of measuring 'distance' in the graph based relational models. In measuring spatial relations the concept of depth is widely used in the literature, meaning the distance between any pair of spatial elements (Hillier and Iida 2005). The three definitions of distance used are: topological distance, the number of turns/steps from one space to another; angular distance, the angular change from one space to another; and the metric distance, the Euclidean distance in metres from one space to another. Different spatial patterns will be generated by assessing the three types of distance from a single street segment. 


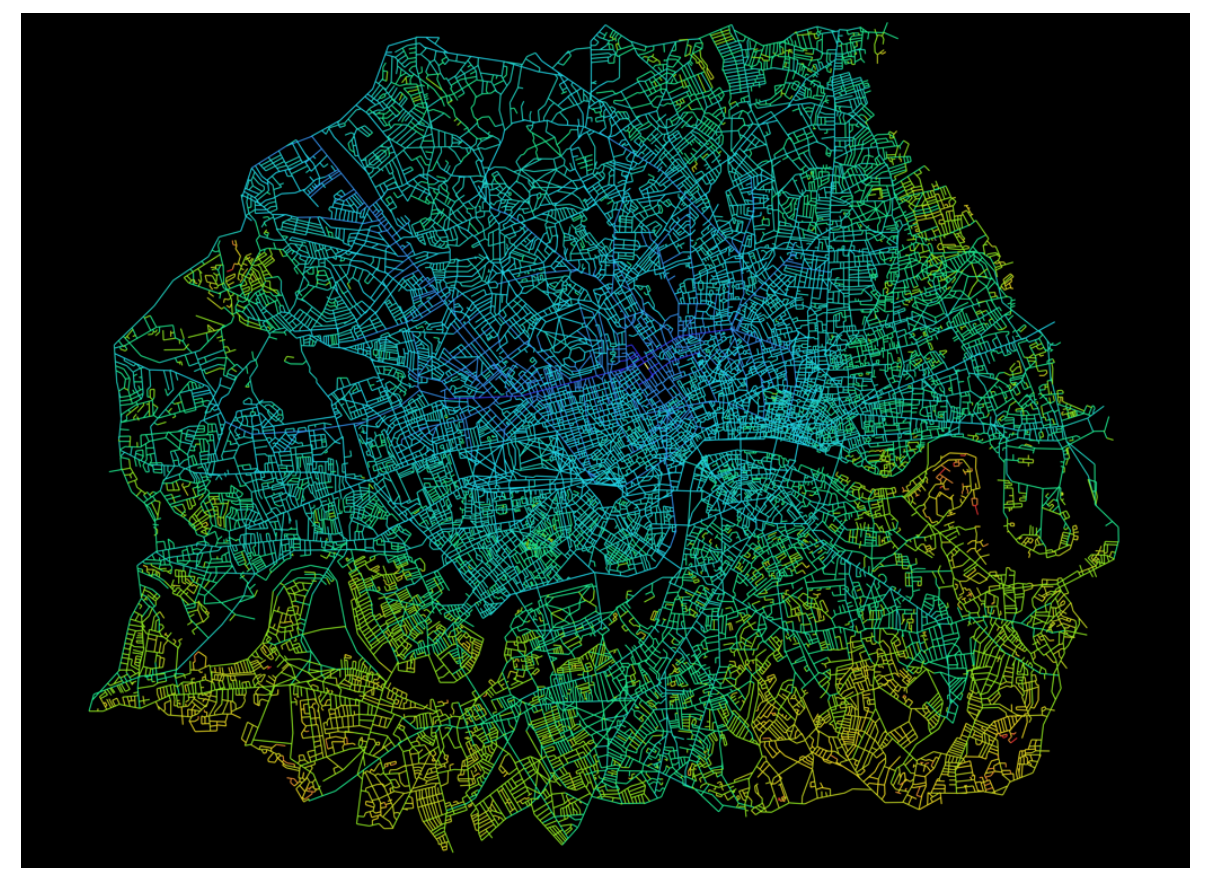

Fig. 2. Angular distance from The Bartlett School of Architecture to every other location in London. Simplified map, 50k segments. Blue represents low distance values, red high.

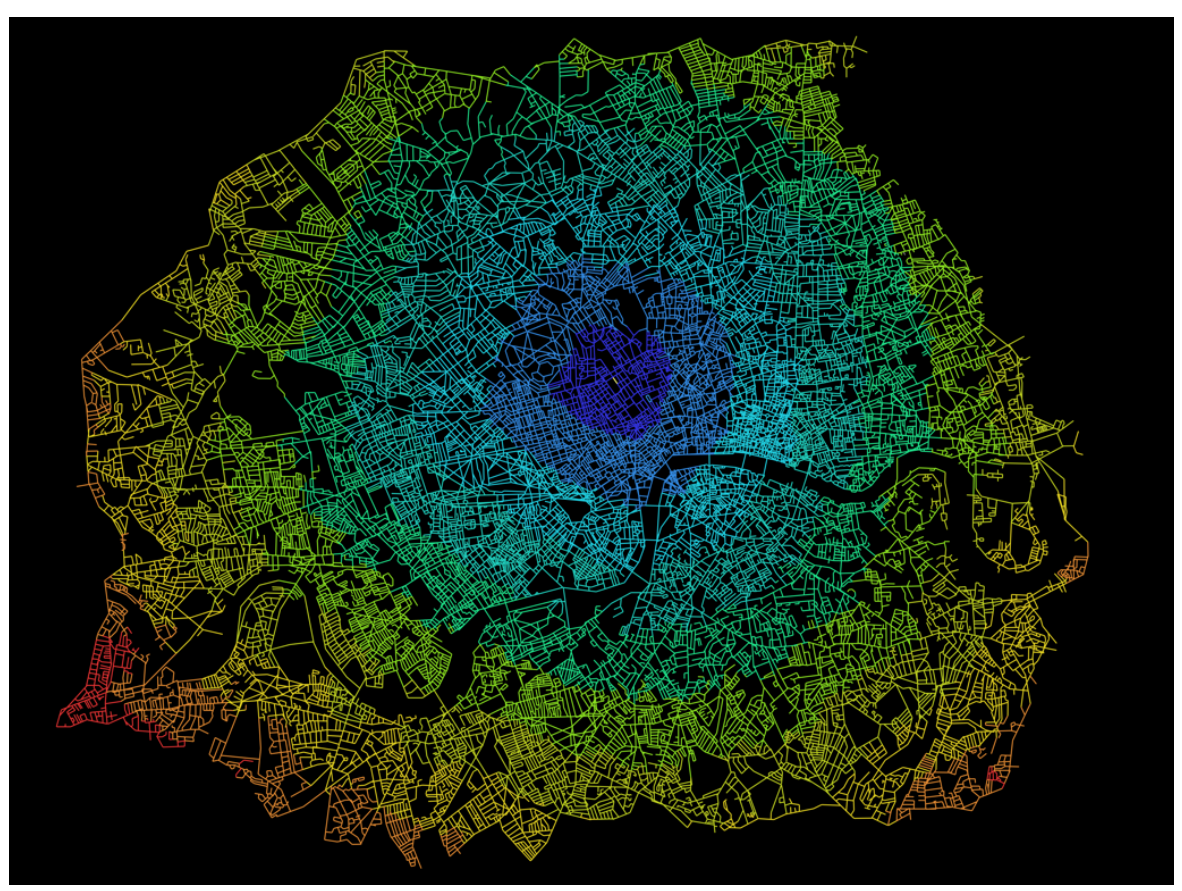


Fig. 3. Metric distance from The Bartlett School of Architecture to every other location in London. Simplified map, 50k segments. Blue represents low distance values, red high.

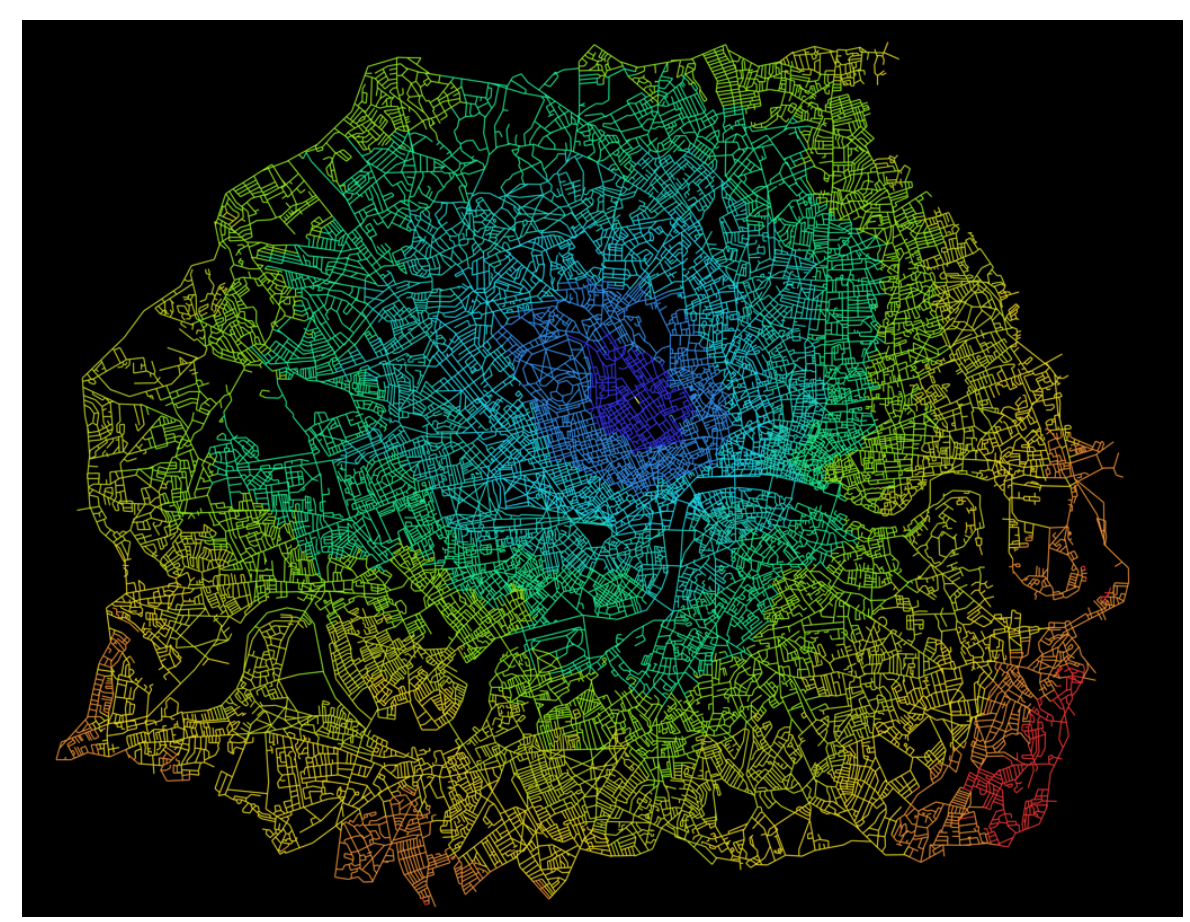

Fig. 4. Topological distance from The Bartlett School of Architecture to every other location in London. Simplified map, 50k segments. Blue represents low distance values, red high.

The figures 2, 3 and 4 depict the different spatial structures when the street network is assessed based on: angular distance (fig. 2), metric distance (fig. 3) and topological distance (fig. 4) from The Bartlett School of Architecture to every other location in London. Blue represents low distance values, red high. We see how certain morphological complexities force areas to be more segregated in different distance definitions (London's street network is simplified for this visualisation; total of 50000 streets).

In the following sections we will decompose our model and present how we can incorporate all three ideas of 'depth' creating a highly detailed multi-dimensional dataset that can provide insights of spatial dynamics beyond what traditional space syntax models could achieve.

\subsection{Local to global - Neighbourhood spatial penetration}

The project started with the dataset design and the aim to create comparative knowledge between the local and global spatial morphology of Great Britain (GB) in a continues feature space, from urban to rural as one thing. Our GB dataset consisted of 4 million 
road segments. Basic cleaning and pre-processing were applied like de-duplication of multiple lanes and non-destructive simplifications. Each straight road or road segment is represented by a single linear element. The completed road network is then read as a graph, with each node corresponding one of the segment lines and connections to each road line it intersects, similar to the space syntax literature (Hillier 2007, Turner 2007). From the dataset we extracted one local subgraph per street segment of a set size $\mathrm{n}$ (n $=200$ in this publication), which ensures that any computational analysis is always made between graphs of equal size (Robles-Kelly and Hancock 2003, Hanna 2009). Starting from street segment $V$ _origin, defined as the centre of the subgraph, we use the angular graph distance measure depicted in figure 2 (Hillier and Iida 2005; Turner, 2007) to calculate the angular distances to all other segments in the graph, where $\mathrm{D}$ (origin, destination) is the angular distance from segment $V_{-}$origin to $V$ _destination. These are then sorted in descending order such that $D(o, d 1)<D(o, d 2)<\ldots<D(o, \ldots)$, and a new subgraph is composed of the closest $n$ (i.e 200 here) segments.

This creates local neighbourhoods of street segments that represent the closest 'angular shortest path' graph, a method not considered in past studies and that traditionally has found strong correspondence in explaining aggregate movement and morphological understanding of built environment by many space syntax studies (Hillier and Iida 2005). Additionally, it creates a scale-agnostic local penetration understanding of the built environment were natural break or significant dissimilarities can be easily identified.

Throughout the project we investigated different values of neighbourhood sizes, $n=$ $50,200,500$ and others, but here we present the findings from local sub areas of size $n$ $=200$ (i.e. the 200 angularly closest streets around a central segment).

\subsection{Graph Spectra}

The graph spectrum of a closest 'angular shortest path' neighbourhood extracted with the method described above consists of the ordered set of eigenvalues of its connectivity matrix. The standard method for deriving the spectral representation begins with an unweighted graph given by a set of nodes $V$ and edges/links $E$, as presented by Luo et al. (2003), Robles-Kelly and Hancock (2003), and Hanna (2012), and can be obtained from an adjacency matrix representing all connections between nodes based on the following algorithm:

$$
A(i, j)=\left[\begin{array}{c}
1 \text { if }(i, j) \in E \\
\text { or } \\
0 \text { otherwise }
\end{array}\right.
$$

Eigenvalues $\lambda$ and eigenvectors $\varphi$ for $A$ are derived: $A=\Phi \Lambda \Phi^{T}$ where the matrix $\Phi=$ $[\varphi 1|\varphi 2| \ldots|\varphi| V \mid]$ contains the eigenvectors as columns and the matrix $\Lambda=\operatorname{diag}(\lambda 1, \lambda 2, \ldots, \lambda|V|)$ contains the eigenvalues as diagonal elements. The spectrum is defined as the set of ordered eigenvalues: $S=\left[\lambda^{1}, \lambda^{2}, \ldots, \lambda^{0}\right]$. At this point, eigenvalues are sorted to form a feature vector by actual value such that $\lambda 1>\lambda 2>\ldots>\lambda|V|$ (Hanna, 2009). 
Graph spectra extracted from topological adjacency matrices have been widely demonstrated in the literature (Luo et al. 2003, Robles-Kelly and Hancock 2003, Hanna 2012, Van Dam and Haemers 2002) to reliably capture differences between the overall topogeometric structure of compared graphs and they are almost unique to a particular spatial graph, especially of the size presented here $(n=200)$.

The first clear distinction of the work presented here and all past studies is that the local sub-graph and subsequent methodology is based on a closest 'angular shortest path' neighbourhood and not a closest topological neighbourhood. Linking back to core space syntax research, we feel that by using this method of neighbourhood extraction to explore micro similarities or differences we created a dataset that more closely encapsulates the embodied understanding of someone's immediate environment that can be captured in higher dimensionality data.

\subsection{Composite 'angular-scalar' graph spectra}

While past studies focus on cities in isolation or small urban areas within a single city and only use the angular deviation between segments to describe the geometry of a topological graph (Hanna, 2009, 2012) our aim is to create a GB-wide cooperative analysis of urban and rural areas together in a continuous space. We see the evaluation of topo-geometric transition between different urban densities and rural towns as the most significant step in understand spatial integration and segregation as a function of natural 'breaks' between areas. This seamless continuity also avoids some parallels to the space syntax 'problem' of edge-effect, when urban networks are artificially cropped from the rest of the dataset (Hillier 2007, Gil 2015, Okabe and Sugihara 2012).

This unified spatial dataset means that graph spectra extracted from a small part of a town in Wales, using past methods, could have similar topological or angular spectra to a graph extracted from a rural area in Cornwall, with the only difference being their relative scale.

To overcome this problem, we propose the use of a new composite 'scale-angular' geometric spectra that can extract high dimensionality data based only on the pure geometric characteristics of the GB's road network. Seeking to encode the angular deviation between street segments and their relative scale create a complete set of geometric data not consigned in the past. Additionally, the fact that the scale is encoded in the spectral model enhances the 'local' to 'global' comparative model significantly.

\section{Angular and scale weighting}

The base process of creating the spectra of a graph described earlier captures the topology only, as the geometry is not represented in the adjacency (connectivity) matrix of a graph.

To create the composite spectral vector we start by extracting the angle weighted spectra with a similar approach to Hanna (2012). The 'continuity' of segments in a straight line is weighted highest (1.0) while values are decreasing with greater angles between the adjacent segments. In all data fields of the binary matrix (1) that represented a connection with a value of 1 and all unconnected nodes with 0 , an updated value for 
connected nodes can be extracted from feeding the adjacent unit vectors of the segments in:

$$
A(i, j)=\left[\begin{array}{c}
\operatorname{acos}\left(\text { ivect } \cdot \mathrm{j}_{\text {vect }}\right) / \pi \text { if }(i, j) \in E \\
\text { or } \\
0 \text { otherwise }
\end{array}\right.
$$

which results in a maximum $A(i, j)=1$ when $i$ and $j$ are part of a single, straight line, and decreasing to zero as the turn angle becomes more acute (Hanna 2012).

We do the same process again for the 'scale' weighted adjacency matrix but this time we substitute the binary value of 1 in (1) with a normalised value of the average length of the two connected segments $(L 1+L 2) / 2.0$, which also represents the standard way of calculating the travelled distance between segments in depthmapX and in essence throughout space syntax research.

\section{Composite spectra}

Finally, we concatenate the two ordered spectra, first the spectra extracted from the angular weighted adjacency matrix followed by the one extracted from the scale weighted matrix. The single composite scale-angular geometric spectra of a local neighbourhood of $n=200$ segments is a vector of 400 values (shape $=[1 \times 400]$ ). This vector represents a single data point in a 400-dimensional space projection.

Starting from the GB data set of 4 million unique road segments (data elements) we created a 'table-looking' dataset with 4 million rows and 400 column, 1.6Billion floating point numbers ( $\sim$ GBytes).

\section{Spectral Integration: topo-geometric similarities and characteristics}

Generating the angle-scale composite spectra has given us an extremely detailed view of the pure geometric configuration of Great Britain's road network. This is particularly significant because angle-scale composite spectra change gently with gradual changes in the geometric configuration of a spatial graph (Zhu and Wilson 2005) making each vector an excellent proxy to quantify morphological differences across different scales and densities. Additionally, spatial graph spectra are nearly unique to a particular graph (Van Dam and Haemers 2002) especially in this composite form and size.

Projecting every spectral vector (with shape $[1,400]$ for $n=200$ closest segments) in a 400-dimensional space creates a data cloud of topo-geometries were the distance between points can characterise the differences in angle-scale morphology between the extracted neighbourhoods (Zhu and Wilson 2005).

Our initial intuition drove us to generate a Euclidian distance integration model of all data point with all other. Understanding the clusters of close proximity spectra we could identify a special form of 'spectral integration' that can be evaluated as 'uniqueness' analysis of spatial morphologies. 
In this model we focused on pure differences in geometry between each neighbourhood and the average difference between all other neighbourhoods and for simplicity we will only look at London's urban network comprised of roughly 300000 segments. In figure 5 purple represents local spectral topo-geometries that are closely situated in this feature space, morphologically very similar to each other. White represent unique morphological structures or 'outliers' in London.

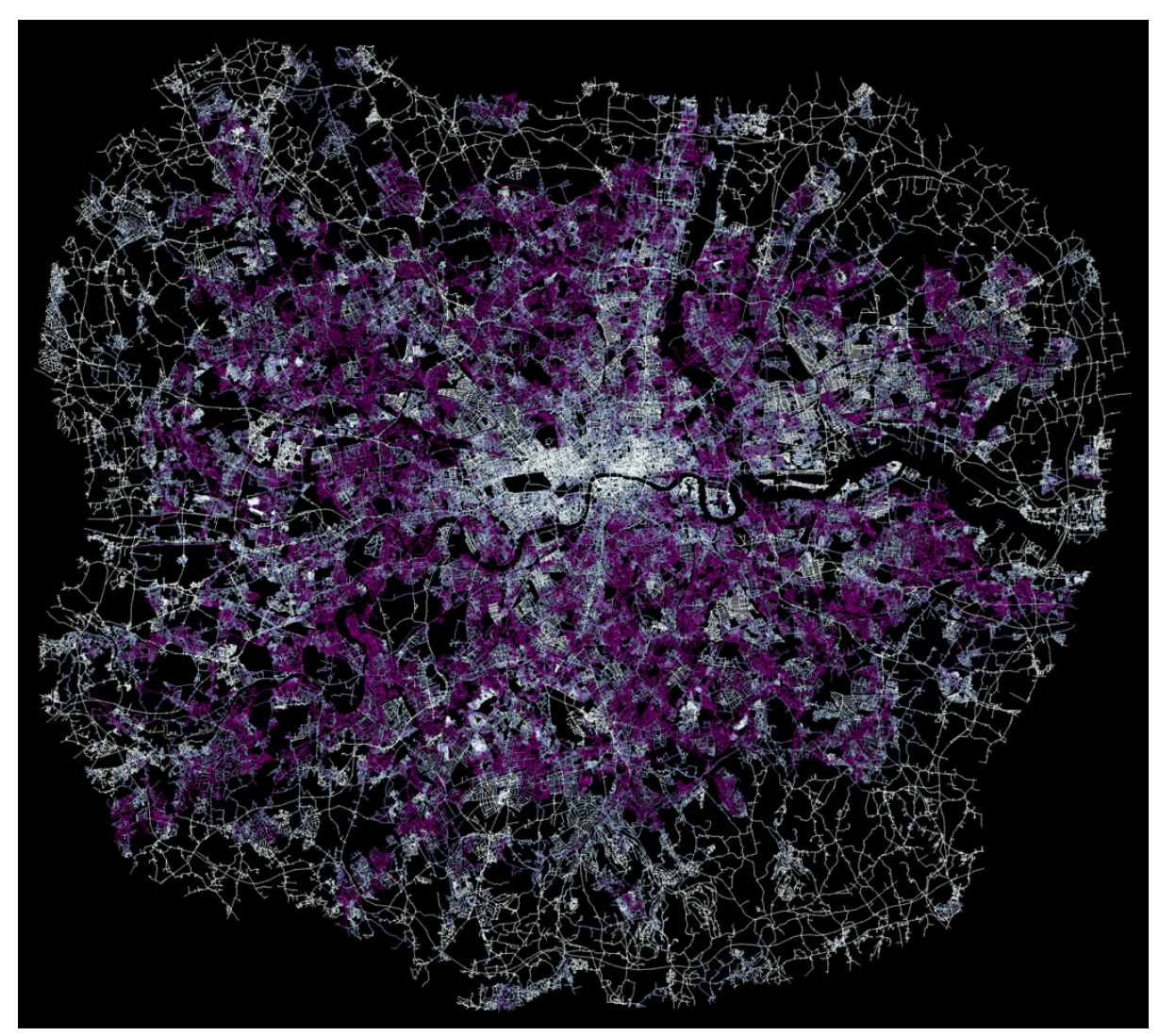

Fig. 5. Composite scale-angular geometric spectra of 300k road segments in London - 'spectral integration - Purple represents local geometries that are closely situated in this feature space, morphological very similar to each other. White represent unique morphological structures.

This model gives a unique perspective in understanding spatial morphologies through graph spectra as the creation of the sub-graphed neighbourhood spectra follows the 'angular shortest path' that is widely regarded to create a link between the immediate spatial morphology of a place and the pedestrian's understanding of it (Hillier 2007). Using these composite angle-scale spectra that depict the internal micro-dynamics of local geometric representations from a human perception enhances traditional network 
theory techniques like space syntax that, unlike graph spectra, generate certain graphs metrics by turning them into aggregate measures of a single scalar value.

Looking at these 'purple' areas of London in figure 5 are what you could call 'typical London looking neighbourhoods', crescents, Victorian houses, in some cases parts of a movie set about London. We also need to note that two different 'white' neighbourhoods are not similar to each other by definition, they can be outliers from different direction in this 400-dimensional space.

\section{$4 \quad$ Unsupervised clustering of composite graph spectra}

Having the angle-scale composite spectra projected into a 400-dimenional feature space that changes gently with gradual changes of the geometry of the neighbourhoods we can easily use an unsupervised clustering model like K-Means (Lloyd, 1957; MacQueen, 1967) to extract similarities. By using an unsupervised clustering algorithm we try to make inferences regarding the morphology of the spectral dataset using only the input vectors (shape $[1,400]$ per local neighbourhood) without referring to any other known, or labelled, data.

K-Means algorithm fed with a point cloud of encoded morphological data can identify a number of clusters through defining the number $\mathrm{k}$, which refers to the number of centroids or clusters you need in the dataset. In our case these will be clusters of similar morphological or spatial characteristics in terms of angularity of the connected segments and scale across the complete GB landscape. In fig 5 you can see an example of how every spectral vector, representing the 200 angularly closest segments from the origin, is allocated to one of the clusters through reducing the in-cluster sum of squares of the spectra. In fig 6 we can see that an unsupervised extraction of a morphological cluster can lousily reassemble the foreground network of London as traditionally described by Hillier (2001 and 2007). Depicting only one cluster we can also observe how different the local spatial penetration patterns can be even though are extracted from similar topo-geometric graph spectra. 


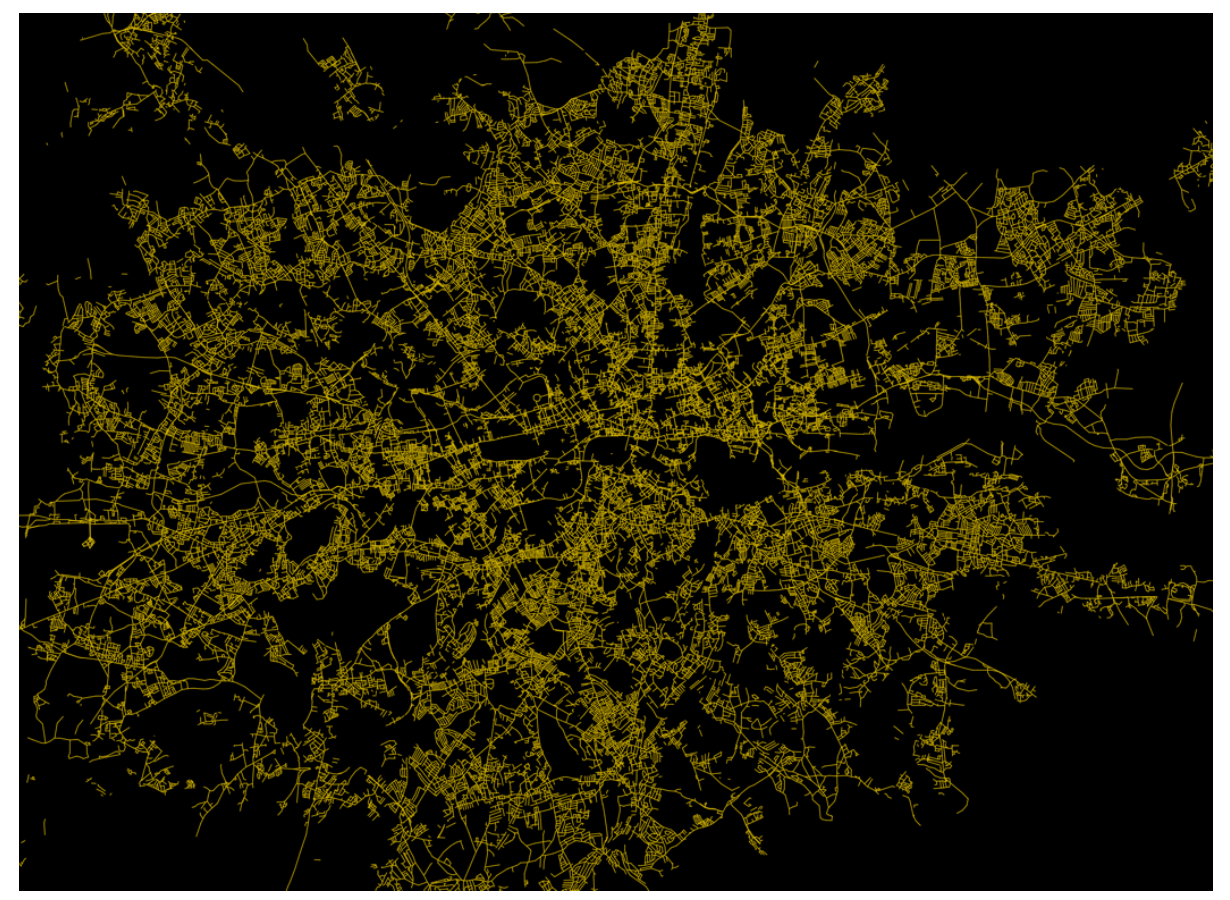

Fig. 6. Initial experiments in London - Composite scale-angular geometric spectra of roughly $300 \mathrm{k}$ road segments projected into a 400-dimensional point cloud and fed into a K-means unsupervised clustering model with $\mathrm{K}=6$. Depicting one cluster only.

Moving to the full GB dataset, figures 7, 8 and 9 depict the K-Means results of 5 angular-scale spectral clusters that all seem to have a very distinct character. Spatial morphologies extracted from a sub-graph with a focus on angular geometry and scale naturally link to timeless ideas from space syntax but also uniquely introduce new propositions for understanding spatial communities and natural breaks in the built environment fabric.

Each of the clusters represent a distinct set of morphological or geometric characteristics. Unsupervised models like these can be used for understanding the natural morphological breaks between wards or apparent communities. Comparing individual subgraphs and the associated composite spectra with can also explore network penetration patterns as depicted in figure 6 . This computational modelling also allows new regional spatial analytics and spatial isolation metrics to be developed. 


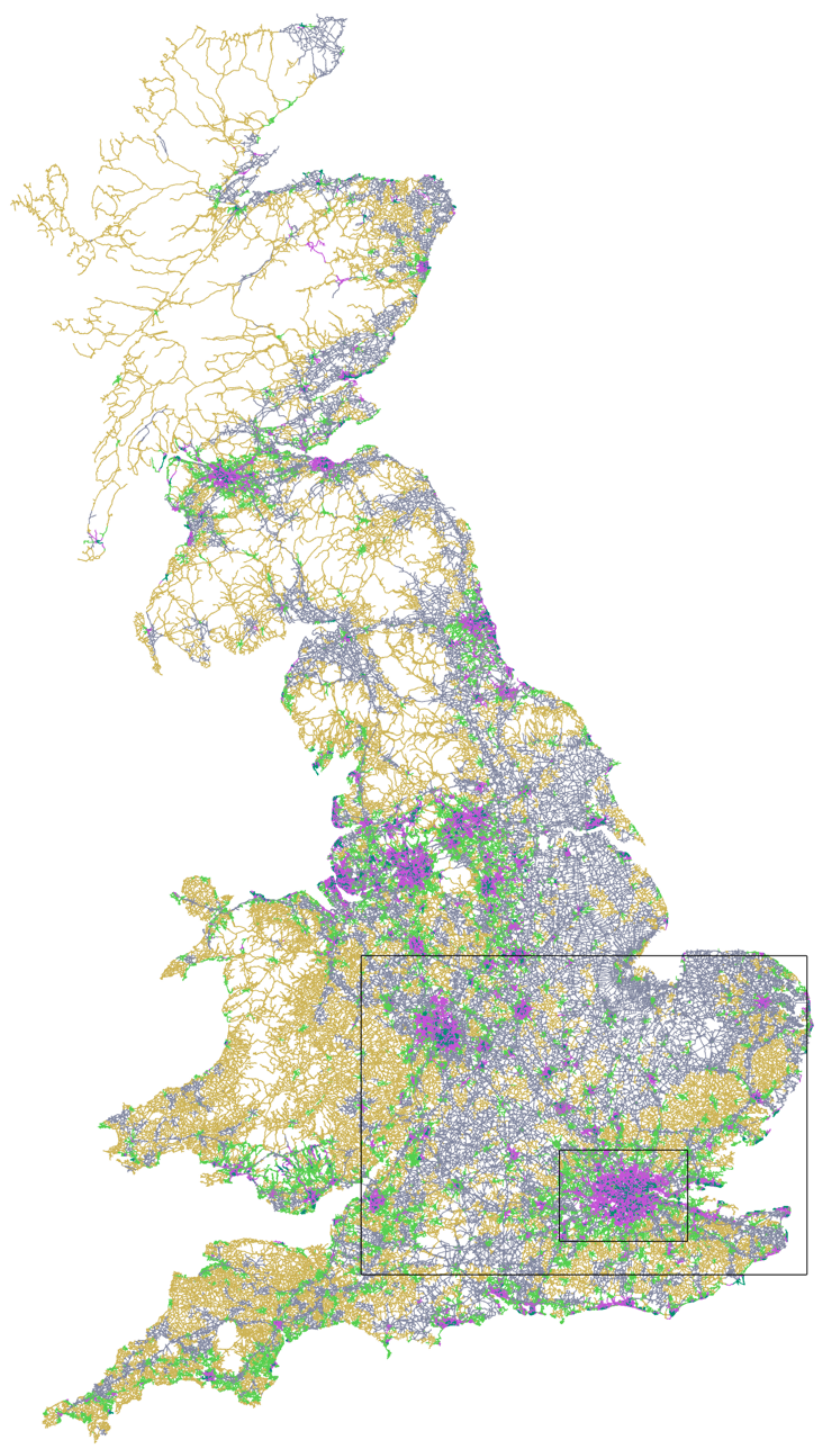

Fig. 7. Great Britain: Composite scale-angular geometric spectra projected into a 400-dimensional point cloud and fed into a $\mathrm{K}$-means unsupervised clustering model with $\mathrm{K}=5$. 


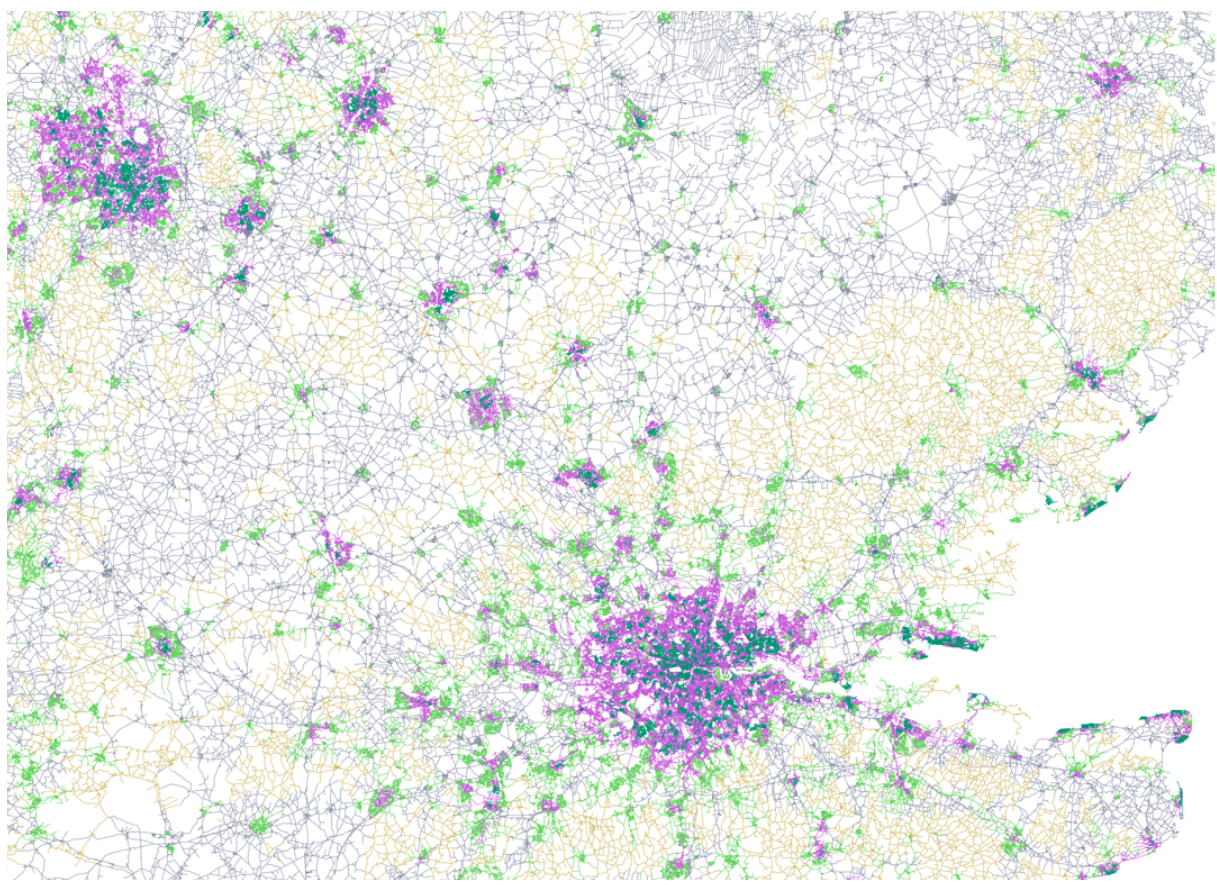

Fig. 8. Composite scale-angular geometric spectra projected into a 400 -dimensional point cloud and fed into a K-means unsupervised clustering model with $\mathrm{K}=5$.

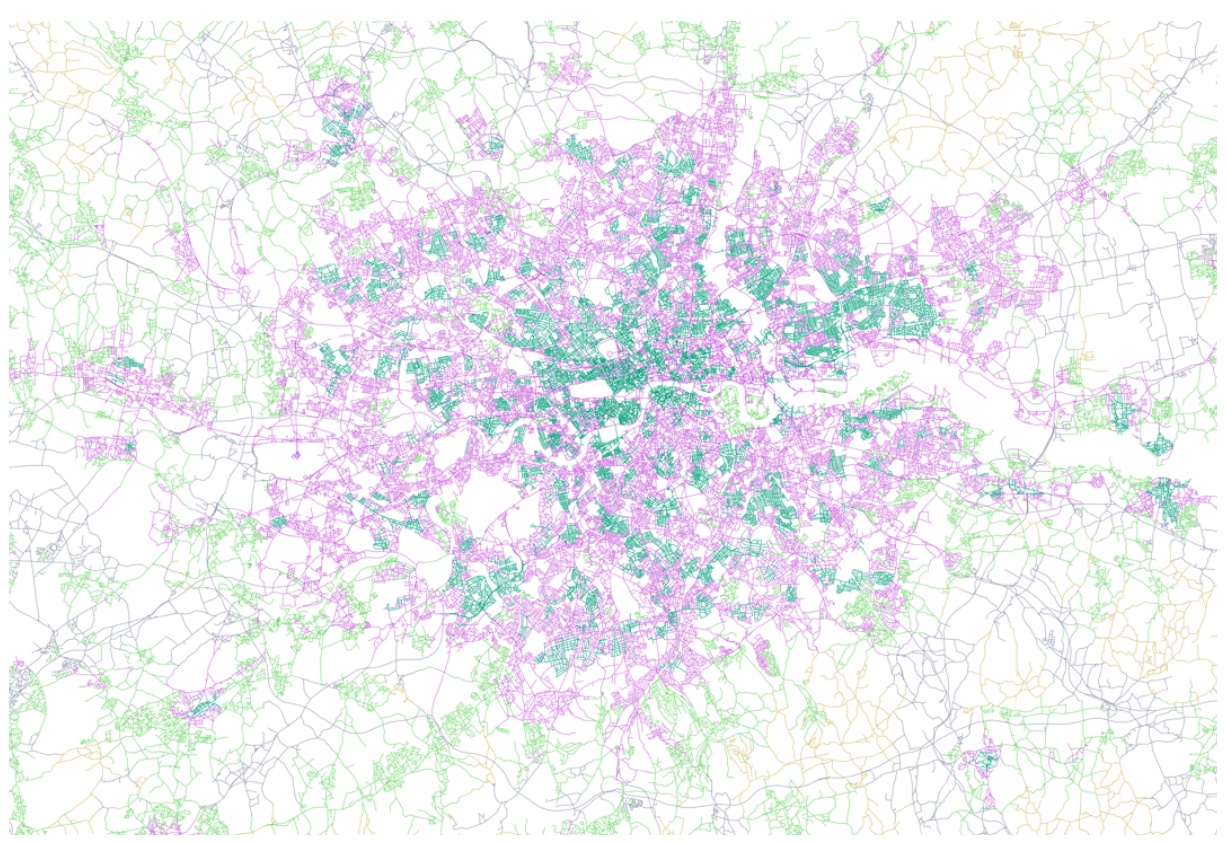


Fig. 9. Composite scale-angular geometric spectra projected into a 400-dimensional point cloud and fed into a K-means unsupervised clustering model with $\mathrm{K}=5$.

\section{Discussions and future}

The work presented ultimately deals with the accelerated paradigm shift in the built environment towards machine intelligence. Traditionally evaluation of spatial networks is done using numerical comparison of spaces with floating point measures such as global and local space syntax measures. The scalar measures fail to retain much of the detail of the spatial topo-geometry and morphology. This is particularly discouraging if the aim is to create data models for subsequent analyses through machine learning techniques. Therefore, we revolved around creating new analyses models and datasets in order to facilitate further research in spatial machine learning.

Gaining insights from our background, the methodology's centre of attention are the three primary space syntax graph modelling concepts, 'angular', 'metric' and 'topological' that create a link between geometry, morphology and human centric perception of the built environment. While looking into existing concepts we identified that in order to create a continues model that uniquely flows between scales and built environment densities we had to develop a new composite graph spectra. Angular change and scale give a complete topo-geometric characterisation of the morphology of the road network. The addition of the "scale" component and the creation of the neighbourhood subgraph following space syntax's 'angular shortest path' can establish a novel GB wide model with comparative power between urban and rural areas.

Having found a way: a) to encode spatial morphology without losing much of the detail of the spatial topo-geometry including scale and angular geometry, b) to create several GB wide models of topo-geometric similarities or dissimilarities of neighbourhoods with the presented form of spectral integration, and finally, c) characterise the topogeometric characteristics by including the differentiation of scale, we aim to use the new dataset in a number of existing new directions.

From an analysis point of view, we currently work on models understanding how the nature of some inter- and intra-regional questions are represented in the new composite spectra. Moreover, detailed mapping of clustered morphological similarities could address challenges of interdependency or spatial isolation of cities, councils or neighbourhoods. Mapping dependencies in a continues form can be a proxy to a numerical fitness model based on the morphology of the road infrastructure.

Finally, from a proposal or design augmentation perspective we focus on how performance-based space syntax analytics can be hybridised with the help of models based on novel machine learning techniques and composite spectra. This is increasingly needed in order to greatly impact research and accelerate practice, local authorities and policy makers. 


\section{Acknowledgements}

The research wouldn't be possible without the support by UK's Engineering and Physical Sciences Research Council (EPSRC) fund EP/M023583/1 and mentoring by Sean Hanna and Bill Hillier at Bartlett's Space Syntax Laboratory.

\section{References}

1. Hillier, B. (2007), Space is the Machine: A Configurational Theory of Architecture. Space Syntax: London, UK. pp.120-121

2. Hillier B and Hanson J (1984) The Social Logic of Space. Cambridge University Press.

3. Turner A 2005, An Algorithmic Definition of the Axial Map, Environment and Planning B: Planning and Design, 32(3) 425-444.

4. Robles-Kelly A and Hancock ER (2003) Edit Distance From Graph Spectra. Proceedings of the ninth IEEE International Conference on Computer Vision (ICCV 2003)

5. Hanna S. 2009. Spectral comparison of large urban graphs, In Koch D, Marcus L and Steen J (eds.) SSS7: Proceedings of the 7th International Space Syntax Symposium. Royal Institute of Technology (KTH), Stockholm, Sweden.

6. Hillier, B, Vaughan, L, (2007) The city as one thing. Progress in Planning , 67 (3) pp. 205230.

7. Turner A (2007) From axial to road-centre lines: a new representation for space syntax and a new model of route choice for transport network analysis, Environment and Planning B: Planning and Design 34(3) $539-555$

8. Varoudis, T. (2012). depthmapX multi-platform spatial network analysis software. OpenSource, http://varoudis.github.io/depthmapX

9. Hillier B, Iida S, (2005) Network effects and psychological effects: A theory of urban movement", in van Nes A (Ed.) 2005, Proceedings of the 5th International Symposium on Space Syntax, TU Delft, Delft, Netherlands, pp 553-564

10. Hanna, S. (2012). Comparative analysis of neighbourhoods using local graph spectra. In Eighth International Space Syntax Symposium. Santiago, Chile.

11. Luo B, Wilson RC and Hancock ER (2003) Spectral embedding of graphs, Pattern Recognition, vol. 36, pp. 2213-2233

12. Van Dam ER and Haemers WH (2002) Spectral Characterizations of Some Distance-Regular Graphs, J. Algebraic Combin. 15, pp. 189-202.

13. Gil, J., 2015, July. Examining "Edge Effects": Sensitivity of Spatial Network Centrality Analysis to Boundary Conditions. In Proceedings of the 10th International Space Syntax Symposium (p. 147).

14. Okabe, A., \& Sugihara, K. (2012). Spatial Analysis Along Networks: Statistical and Computational Methods. John Wiley \& Sons. p.41

15. Zhu P and Wilson RC (2005) A Study of Graph Spectra for Comparing Graphs. British Machine Vision Conference 2005.

16. Lloyd, S. P. (1957). Least squares quantization in PCM. Technical Report RR-5497, Bell Lab, September 1957.

17. MacQueen, J. B. (1967). Some methods for classification and analysis of multivariate observations. In L. M. Le Cam \& J. Neyman (Eds.), Proceedings of the fifth Berkeley symposium on mathematical statistics and probability (Vol. 1, pp. 281-297). California: University of California Press. 
18. Hillier B., (2001), 'A Theory of the City as Object: Or, how spatial laws mediate the social construction of urban space'. 